\title{
NONCUT POINTS AND MODIFIED COMPACTNESS CONDITIONS
}

\author{
R. M. STEPHENSON, JR. ${ }^{1}$
}

1. Introduction. This paper presents generalizations of the two basic theorems on noncut points in $T_{1}$ continua given in [7], the generalizations consisting primarily in weakening compactness to rather more esoteric conditions. The results cover a considerable number of classes of spaces, defined as minimal or complete with respect to certain properties, all of which have been studied, for different purposes, at some leng th in the existing literature.

The terminology used is, in general, the same as that in [7]. We shall denote by $C(X)$ the set of continuous real valued functions defined on a space $X$, and we shall denote by $L(X)$ the set of functions in $C(X)$ which map $X$ into $[0,1]$. Two points $x, y$ in $X$ are completely separated if there is a function $f$ in $C(X)$ with $f(x) \neq f(y) ; X$ is called completely Hausdorff if every pair of distinct points of $X$ are completely separated. If for some $z$ in $X, X-\{z\}=\mathrm{A} \cup B$, separated in $X-\{z\}$, with $x$ in $A$ and $y$ in $B$, then $x$ and $y$ are said to be point separated.

A filter base on a space $X$ is said to be an open filter base if the sets belonging to it are open subsets of $X$. An open filter base $R$ is regular if each set $R \in R$ contains the closure of some set $S \in R$. An open filter base $\mathcal{C}$ is completely regular if for every $C \in \mathcal{C}$ there exist a set $D \in \mathcal{C}$ and a function $f \in L(X)$ such that $f$ vanishes on $D$ and equals 1 on $X-C$. A filter base $\mathcal{F}$ is fixed if $\cap \mathcal{F} \neq \varnothing$.

On any space $X$ each of the following is a necessary condition for compactness.

(A) Every regular filter base on $X$ is fixed.

(B) Every completely regular filter base on $X$ is fixed.

We define $a$ to be the class of nondegenerate connected $T_{1}$ spaces $X$ for which $A$ holds and $B$ to be the class of nondegenerate connected $T_{1}$ spaces $X$ for which $B$ holds and every pair of point separated points of $X$ are completely separated.

a consists of the nondegenerate connected $T_{1}$ spaces which have the property " $R(i)$ " studied in [9]. B consists of the nondegenerate

Received by the editors June 17, 1968.

1 This research was partially supported by a National Science Foundation grant GP-6220. A portion of this paper is a part of a doctoral dissertation at Tulane University, directed by Professor M. P. Berri. 
connected $T_{1}$ spaces which have a property that is slightly more general than the property "completely Hausdorff-complete" studied in [12]. Immediate proofs, based on known characterization theorems, show that $a$ contains the nondegenerate connected $T_{1}$ spaces which are compact, minimal Hausdorff, absolutely closed, nonvacuously $H(i i), H(i)$, minimal Urysohn, Urysohn-closed, nonvacuously $U(i i)$, $U(i)$, minimal regular, regular-closed, nonvacuously $R(i i)$, or $R(i)$ (see [1]-[3], [6], [8]-[10], and [12]). It follows from the results in $[1]$ and $[12]$ that the class $B$ contains the nondegenerate connected Hausdorff spaces which are compact or completely Hausdorffcomplete.

\section{The class $Q$.}

Lemma 2.1. Let $X$ be a connected $T_{1}$ space, let $x \in X$, and suppose that $X-\{x\}=A \cup B$, separated. Then $A$ and $B$ are open in $X, \bar{A}=A \cup\{x\}$, $\bar{B}=B \cup\{x\}$, and $\bar{A}$ and $\bar{B}$ are connected.

Leмma 2.2. Let $X$ be a connected $T_{1}$ space, and suppose that there exist a subset $A$ of $X$ and sets $U(a), V(a) \subset X, a \in A$, which have the following properties: for each $a \in A, X-\{a\}=U(a) \cup V(a)$, separated; for all $a, b \in A, U(a) \subset U(b)$ or $U(a) \supset U(b)$. Then one of the following holds.

(i) $\{U(a) \mid a \in A\}$ is fixed.

(ii) $\{U(a) \mid a \in A\}$ is a regular filter base on $X$.

Proof. If there exists $c \in A$ such that for all $a \in A-\{c\}, U(c)$ $D U(a)$, then (i) holds. Suppose that for all $a \in A$ there exists $c \in A-\{a\}$ such that $U(a) \supset U(c)$. Then an argument based on Lemma 2.1, similar to one in the proof of Theorem 2-18 in [7], shows that for each $a$ there exists $c$ so that $U(a) \supset U(c) \cup\{c\}=[U(c)]^{-}$. Thus (ii) holds.

THEOREM 2.3. If $S \in Q$, then $S$ has at least two noncut points.

Proof. The subcollection $\left\{U_{x_{\alpha}}\right\}$ in the proof of Theorem 2-18 in [7] satisfies the hypothesis of Lemma 2.2, so the requirement that $S$ be compact can be weakened to the property $A$.

Lemma 2.4. Let $X$ be a space which has property $A$. If $V$ is an open subset of $X$ and $x \in X-V$ with $\bar{V}=V \cup\{x\}$, then $V \cup\{x\}$ has property $A$.

Proof. Let $R$ be a regular filter base on $V \cup\{x\}$, and suppose that $x \notin \cap R$. Then there is a set $R \in R$ such that $x \in \bar{R}$. The filter base $R^{\prime}=\{S \in R \mid S \subset R\}$ is a regular filter base on $X$. Thus $\varnothing \neq \cap \Omega^{\prime} \subset \cap R$. 
A connected space $S$ is irreducibly connected about a set $A \subset S$ provided that no proper connected subset of $S$ contains $A$.

Theorem 2.5. If $S \in Q$, then $S$ is irreducibly connected about its set of noncut points.

The proof is an obvious modification of the proof of Theorem 2-19 in [7].

EXAmple 2.6. If in [3] one starts with the "long interval" instead of the set of ordinals $\leqq \Omega$ and the one-point compactification of $[0, \infty)$ instead of the set of ordinals $\leqq \omega$, then the same construction as the one in [3] yields a connected, locally connected, minimal regular space $Y$ which consists entirely of noncut points.

We omit the proof, since it is long, but essentially the same as the one in [3].

EXAMPLE 2.7. Let $R$ be the irrational numbers in $[0,1], X_{0}$ $=R \times\{0\}, X_{i}=([0,1]-R) \times\{i\}, i=1,2$, and $X=X_{0} \cup X_{1} \cup X_{2}$. The topology for $X$ is generated by intersections with $X$ of sets of the form $A \times\{0,1,2\}, A \times\{1\}$, and $A \times\{2\}$, where $A$ is open in $[0,1]$. In [5] Herrlich proves that $X$ is minimal Hausdorff but not second category. One can also see that $X$ is connected, and that its cut points and noncut points are $X_{0}$ and $X_{1} \cup X_{2}$.

EXAMPLE 2.8. The noncompact minimal Hausdorff space in $[10]$ is connected and consists entirely of noncut points.

These spaces are not countably compact or completely Hausdorff. The following noncompact space has both properties.

EXAMPLE 2.9. Let $Y$ be the "long interval," with the weakest topology stronger than the order topology which contains the complement of the ordinals less than $\Omega$. Then [12] $Y$ is countably compact, completely Hausdorff, and absolutely closed. $Y$ also belongs to $a$ and is irreducibly connected about its first and last points.

\section{The class $B$.}

Lemma 3.1. Let $X$ be a space with property $B$ and $f$ a continuous mapping of $X$ into a space $Y$. Then $f(X)$ has property $B$.

Lemma 3.2 (Banaschewski). A completely regular space with property $B$ is compact.

This is an immediate consequence of the principal result in [1].

Lemma 3.3. Let $V$ be an open subset of a space $X$ and $x$ a point of $X-V$ such that $V \cup\{x\}=\bar{V}$. If $X \in ß$, then $\bar{V} \in ß$. 
Proof. Let $R$ be a completely regular filter base on $V \cup\{x\}$, and suppose that $x \notin \cap R$. Let $\mathcal{R}^{\prime}=\{R \in R \mid x \notin R\}$, and consider a set $C \in R^{\prime}$. There exist $D \in R^{\prime}$ and $f \in L(V \cup\{x\})$ such that $f(D)=0$ and $f((V \cup\{x\})-C)=1$. Define $g: X \rightarrow[0,1]$ by $g(t)=1$ if $t \notin C$ and $g(t)=f(t)$ otherwise. Then $g$ is continuous on $X, g(D)=0$, and $g(X-C)=1$. Thus $R^{\prime}$ is a completely regular filter base on $X$, so $\varnothing \neq \cap \mathbb{R}^{\prime} \subset \cap R$.

Since $X \in B$ and every pair of point separated (with respect to $\bar{V}$ ) points of $\bar{V}$ are point separated with respect to $X$, every pair of point separated points of $\bar{V}$ are completely separated.

If $X$ is any topological space, then one can associate with $X$ a completely regular space $w X$ and a continuous mapping $c$ of $X$ onto $w X$ such that $C(X)=\{g \circ c \mid g \in C(w X)\}[4$, p. 41]. In case $X$ is completely Hausdorff, $w X$ and $c$ can be chosen so that $w X$ has the same points as those of $X$ and $c=$ the identity mapping.

Lemma 3.4. Let $X \in$ B. If $x$ is a cut point of $X$, then $c(x)$ is a cut point of $w X$.

Proof. Suppose that $X-\{x\}=A \cup B$, separated. Then $A$ and $B$ are open, and $\bar{A}=A \cup\{x\}$ and $\bar{B}=B \cup\{x\}$ have property $B$ by Lemma 3.3. Thus $c(\bar{A})$ and $c(\bar{B})$ are closed subsets of $w X$ by Lemmas 3.1 and 3.2. Since each pair of point separated points of $X$ are completely separated, $c(\bar{A}) \cap c(\bar{B})=\{c(x)\}$. Therefore, $w X-\{c(x)\}$ $=(c(\bar{A})-\{c(x)\}) \cup(c(\bar{B})-\{c(x)\})$ is a decomposition of $w X-\{c(x)\}$ into open sets.

Theorem 3.5. Let $X \in B$. Then $X$ has at least two noncut points.

Proof. If $X$ has at least one cut point, then $w X$ is a nondegenerate Hausdorff continuum and has at least two noncut points $a$ and $b$. The points in $c^{-1}(a)$ and $c^{-1}(b)$ are noncut points of $X$.

Corollary 3.6. Let $X$ be a connected completely Hausdorf-complete space (see [12]). Then $X$ and wX have the same noncut points.

Proof. A completely Hausdorff-complete space has property $B$ [12], so for every noncut point $a$ of $w X, a=c^{-1}(a)$ is a noncut point of $X$. Conversely, it follows from the continuity of $c$ that every noncut point of $X$ is a noncut point of $w X$.

Theorem 3.7. If $X \in ß$, then $X$ is irreducibly connected about its set of noncut points.

Proof. The proof is an appropriate modification of the proof of Theorem 2.5. 
If $X$ is any one of the spaces in Examples 2.6-2.9, one can prove that every pair of point separated points of $X$ are completely separated. Since, clearly, property $A$ is a sufficient condition for property $B$ on any space, it follows that the spaces in Examples 2.6-2.9 belong to $B$.

ExAmple 3.8. Let $X=[0,1]$ and $v$ be the usual topology on $X$, and choose a collection of sets $X(n), n=1,2, \cdots$, which have the following properties: for each $n, X(n)$ is a dense subset of $(X, \mathcal{U}) ; X(i) \cap X(j)$ $=\varnothing$ whenever $i \neq j ; X=U\{X(n)\}$. Denote by $w$ the topology on $X$ generated by $V \cup\{X(2 n-1) \cup X(2 n) \cup X(2 n+1) \mid n=1,2, \cdots\}$ $\cup\{X(2 n-1) \mid n=1,2, \cdots\}$. A proof is given in [11] that $w(X, w)$ $=(X, V)$ and that there exists a countable regular filter base on $(X, W)$ which is not fixed. According to [1], a completely Hausdorff $Y$ has property $B$ if and only if $w Y$ is compact. Thus $(X, w)$ $\in B-Q$.

4. Nondegenerate $T_{1}$ continua and the classes $a$ and $B$. There are, not surprisingly, several important ways in which noncompact connected spaces with property $A$ or $B$ fail to be as well behaved as the compact ones.

EXAMPLE 4.1. If $U$ is an open subset of a countably compact completely Hausdorff space $Y \in Q \cap \beta$ and $C$ is a component of $U$, then $\bar{U}-U$ need not contain any limit points of $C$. Using the notation in [12, Example 5], let $U=Y-\Omega^{\prime}$ and $C=\{\Omega\}$.

EXAMPLE 4.2. An absolutely closed completely Hausdorff space can have a quasi-component that is not a component.

Let $Y=[0,1], Z=\{0\} \cup\{1 / n \mid n=1,2, \cdots\}, X=Y \times Z$, and let $v$ denote the usual topology on $X$. We write $(a, b)^{\prime}$ for the point $(a, b)$ and $(a, b)$ for the open interval $(a, b)$. Let $W$ be the topology on $X$ generated by $\left\{V \mid V \in \mathcal{V}\right.$, or $V=\left\{(1,0)^{\prime}\right\} \cup((a, 1] \times((0, b) \cap Z))$ for some $a, b$ in $(0,1)\} .(X, W)$ is an absolutely closed completely Hausdorff space, the quasi-component of $(0,0)^{\prime}$ in $(X, W)$ is $Y \times\{0\}$, and the component of $(0,0)^{\prime}$ in $(X, W)$ is $\left\{(a, 0)^{\prime} \mid a \in[0,1)\right\}$.

The proof that $(X, W)$ is absolutely closed and completely Hausdorff is similar to the proof in [12, Example 5]. Proofs of the other statements are straightforward.

REMARK 4.3. According to Theorem 2.4 of [13], no noncompact connected, locally connected space is irreducibly connected about a compact subset. Thus the noncompact spaces covered by Theorem 2.5 or 3.7 will not be locally connected unless they have infinitely many noncut points.

REMARK 4.4. If a space $X \in Q$ is minimal Hausdorff (minimal 
Urysohn, minimal regular) and has two noncut points, then it follows from Theorems $11.5-11.7$ of [13] that $X$ is an ordered continuum.

EXAmple 4.5. The following is a sequentially compact, noncompact, minimal Hausdorff space $X \in Q$ which has three noncut points.

Let $\Omega^{\prime}$ be as in Example 4.1, let $R$ be the nonnegative real numbers, and let $Z$ be the lexicographically ordered space $\Omega^{\prime} \times R$. Choose two new points $a_{1}, a_{2}$ and let $X=Z \cup\left\{a_{1}, a_{2}\right\}$, topologized as follows: if $V$ is open in $Z$, then $V$ is open in $X$; neighborhoods of $a_{1}$ and $a_{2}$ are any sets of the form

$$
\begin{array}{llll}
V^{\alpha}\left(a_{1}\right)=\left\{a_{1}\right\} \cup\{(\beta, r) \in Z \mid \beta>\alpha & \text { and } & 0<r<1\}, & \alpha \in \Omega^{\prime}, \\
V^{\alpha}\left(a_{2}\right)=\left\{a_{2}\right\} \cup\{(\beta, r) \in Z \mid \beta>\alpha & \text { and } & r>1\}, & \alpha \in \Omega^{\prime} .
\end{array}
$$

Proof. $Z$ is the long line, so $X$ is sequentially compact. Since $\Omega^{\prime} \times\{0\}$ is a closed, noncompact subset of $X, X$ is not compact. $X$ is obviously Hausdorff.

To prove that $X$ is minimal Hausdorff, it suffices (see, e.g., [2], or pp. 167-168 in Bourbaki's Topologie gênérale) to prove that (a) $X$ is absolutely closed and (b) the family $\{\operatorname{Int}(\mathrm{Cl} V) \mid V$ is an open subset of $X\}$ is a base for the topology on $X$. The proof of (a) is similar to the proof needed for Example 4.2. (b) is clear except possibly at $a_{1}$ and $a_{2}$. For each $a_{i}$ and $\alpha$, one can show that $V^{\alpha}\left(a_{i}\right)=\operatorname{Int}\left(\mathrm{Cl} V^{\alpha}\left(a_{i}\right)\right)$.

Proofs of the other statements are straightforward.

We conclude the paper with an example of a compact space $K \in Q-\beta$.

EXAMPLE 4.6. Let $U$ be any nondegenerate countable connected $T_{1}$ space, let $P$ be the product space $U \times\{1,2\}$, and let $K=P \cup\{\pi\}$, topologized as follows: a set $V \subset K$ is open if (a) $V \cap P$ is open in $P$ and (b) if $\pi \in V$, then $K-V$ is finite.

Since $C(U)$ contains only constant functions, no two points of $K$ are completely separated. On the other hand, any points $(u, 1) \in K$ and $(v, 2) \in K$ are point separated.

\section{REFERENCES}

1. B. Banaschewski, On the Weierstrass-Stone approximation theorem, Fund. Math. 44 (1957), 249-252.

2. - Über zwei Extremaleigenschaften topologischer Räume, Math. Nachr. 13 (1955), 141-150.

3. M. P. Berri and R. H. Sorgenfrey, Minimal regular spaces, Proc. Amer. Math. Soc. 14 (1963), 454-458.

4. L. Gillman and M. Jerison, Rings of continuous functions, Van Nostrand, Princeton, N. J., 1960.

5. H. Herrlich, Nicht alle $T_{2}$-minimalen Räume sind von 2. Kategorie, Math. Z. 91 (1966), 185. 
6. - $-T_{\nu}$-Abgeschlossenheit und $T_{\nu}$-Minimalität, Math. Z. 88 (1965), 285-294.

7. J. G. Hocking and G. S. Young, Topology, Addison-Wesley, Reading, Mass., 1961.

8. C. T. Scarborough, Minimal Urysohn spaces, Pacific J. Math. 27 (1968), 611-617.

9. C. T. Scarborough and A. H. Stone, Products of nearly compact spaces, Trans. Amer. Math. Soc. 124 (1966), 131-147.

10. N. Smythe and C. A. Wilkins, Minimal Hausdorff and maximal compact spaces, J. Austral. Math. Soc. 3 (1963), 167-171.

11. R. M. Stephenson, Jr., Pseudocompact spaces, Trans. Amer. Math. Soc. 134 (1968), 437-448.

12. - Spaces for which the Stone-Weierstrass theorem holds, Trans. Amer. Math. Soc. 133 (1968), 537-546.

13. R. L. Wilder, Topology of manifolds, Amer. Math. Soc. Colloq. Publ., vol. 32, Amer. Math. Soc., Providence, R. I., 1949.

TUlane UNIVERSity AND

University of North Carolina at Chapel Hill 